\title{
Three-dimensional limit analysis of rigid blocks assemblages. Part I: Torsion failure on frictional interfaces and limit analysis formulation
}

\author{
A. Orduña *, P.B. Lourenço \\ Department of Civil Engineering, University of Minho, Azurém, 4800-058 Guimarães, Portugal
}

Received 6 July 2004; received in revised form 8 February 2005

Available online 17 March 2005

\begin{abstract}
The governing equations of plastic torsion of, arbitrarily shaped, frictional interfaces are established and a general solution is proposed. The case of rectangular interfaces is used to study the interactions of torsion strength with bending moments and shear forces. In order to solve limit analysis problems, a piecewise linear approximation of the yield function for rectangular interfaces is proposed. A model for the limit analysis of three-dimensional block assemblages interacting through no-tension frictional interfaces is presented including the proposal for the torsion failure mode. This model takes into account non-associated flow rules and limited compressive stresses at the interfaces.
\end{abstract}

(c) 2005 Elsevier Ltd. All rights reserved.

Keywords: Limit analysis; Non-associated flow rule; Rigid block; Plastic torsion

\section{Introduction}

Masonry has a low tensile strength and this failure mode is quasi-brittle; therefore, unreinforced and unconfined masonry elements, as those of ancient buildings, are prone to extensive cracking. At failure, these cracks render the structure as a set of rigid blocks rocking and sliding between them. The cracks represent the rocking and sliding interfaces between blocks. For this reason, limit analysis of rigid block assemblages interacting through no-tension, frictional interfaces is a valuable model for ancient masonry structures.

Concepts directly related with the modern limit analysis theory were used centuries ago by renown thinkers as Hooke, Coulomb or Poleni in the assessment of masonry structures. Heyman (1966) established the

\footnotetext{
${ }^{*}$ Corresponding author. Present address: Faculty of Civil Engineering, University of Colima, km 9, Colima-Coquimatlán way, 28400, Mexico. Tel./fax: +52 3123161167.

E-mail address: aord@ucol.mx (A. Orduña).
} 
hypotheses upon which limit analysis is applicable to masonry structures. These hypotheses can now be restated as follows: (1) The limit load occurs at small overall displacements. This hypothesis is generally reasonable; nevertheless, it must be revised for each particular case. (2) Masonry has no tensile strength. The low tensile strength and the quasi-brittle tensile failure of masonry justify this assumption. (3) The shear failure at the interfaces is perfectly plastic, which is confirmed by experimental results (Lourenço and Ramos, 2004). (4) Hinging at an interface occurs for a compressive force independent from the rotation. This assumption might be questioned if crushing occurs, but crushing behaviour seems to have minor significance in the response of historical masonry structures.

The fact that sliding in dry interfaces occurs with almost zero dilatancy (in this paper the dilatancy is assumed equal to zero) renders it as a non-associated flow mode. This violates one of the hypotheses of classic limit analysis theory and requires the use of a mixed formulation, i.e. the static and kinematic approaches cannot be separated. A more fundamental result is that under non-associated flow rules, a multiplicity of solutions can exist for limit analysis problems. Previous authors addressed this issue, namely Begg and Fishwick (1995), which presented a formulation for non-associated limit analysis of two-dimensional voussoir arches with infinite compressive strength. Latter, Baggio and Trovalusci (1998) presented a formulation for three-dimensional, non-associated limit analysis of rigid block assemblages, which is general in the sense that it does not imply assumptions about the arrangement of blocks and interfaces. Nevertheless, the threedimensional examples in the cited paper are solved for associated flow rules only and the authors recognise the difficulties of solving the non-associated version of the problem. These authors proposed to minimise the load factor as a way to obtain the safest solution. Recent developments in the non-linear mathematical programming field have permitted to solve efficiently the Mixed Complementarity Problem (MCP) arising from the non-associated flow limit analysis problem (Ferris and Tin-Loi, 2001). Nevertheless, it is found that minimising the load factor can conduct to severe underestimations of the collapse load.

Orduña and Lourenço (2003) presented a general, two-dimensional formulation of the rigid blocks limit analysis problem where it is possible to limit the compressive stresses at the interfaces and a tie element is included for strengthening design. Extending the compatibility and equilibrium requirements from two to three dimensions is relatively straightforward. Nevertheless, in the extension of the yield conditions and flow rules, apart from the hinging and sliding failure modes, also the twisting mode must be included. This mode presents strong interactions between the torsion strength and the other generalised stresses. The torsion failure on arbitrarily shaped frictional interfaces is a topic poorly studied. In fact, only the work of Goyal et al. (1991) was found in the literature. In the work previously referenced of Baggio and Trovalusci (1998) a very simple torsion model was included in the three-dimensional limit analysis formulation, where the interactions of the torsion strength with the other generalised stresses were neglected.

In this paper, the equations governing the plastic torsion of frictional interfaces are presented and a general solution for arbitrarily shaped interfaces is proposed. The particular case of rectangular interfaces is studied in detail, aiming at assessing the interactions of the torsion strength with the shear forces and bending moments acting on the interface. A piecewise linear approximation to the yield function and flow rule of rectangular interfaces is proposed in order to be included in a limit analysis formulation. A complement to this proposal is the hinging yield function, which takes into account a limited compressive stress at the interface. The complete limit analysis formulation for rigid block assemblages is then presented. In the second part of this paper (Orduña and Lourenço, 2005), a novel solution procedure is proposed and validation examples are presented and discussed.

\section{Plastic torsion on frictional interfaces}

Consider an arbitrary shaped interface $\mathscr{S}$ between two bodies with infinite strength, as the illustrated in Fig. 1. This interface has boundary $\mathscr{B}$. The cohesion-less Coulomb's law governs the interface failure, 


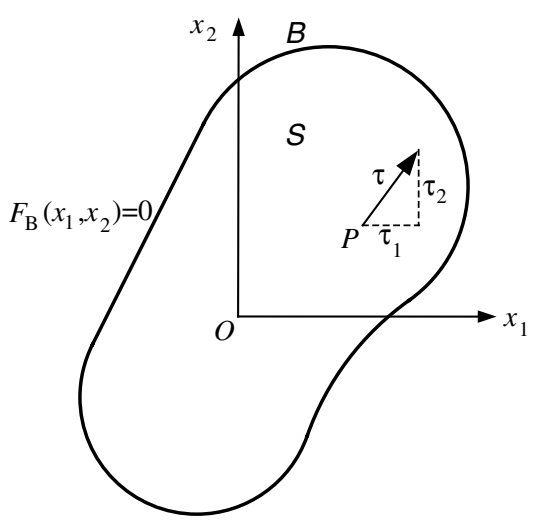

Fig. 1. Interface subject to torsion.

although the inclusion of non-zero cohesion is straightforward. The interface is supposed to represent a dry masonry joint, therefore, it has no tensile strength. The interface is subjected to a compressive force and to a torsion moment. The origin of the coordinate system is located at the centre of plastic torsion, $O$, of the interface. This coordinate system has axes $x_{1}$ and $x_{2}$ on the interface plane, and $x_{n}$ in the normal direction, forming a right-handed system (the axis $x_{n}$ points outwards the plane shown in Fig. 1). The unitary vectors along the coordinate axes $x_{1}, x_{2}$ and $x_{n}$ are $\vec{s}_{1}, \vec{s}_{2}$ and $\vec{n}$, respectively. The shear stress component $\vec{\tau}$ at a point $P$ has components $\tau_{1}$ and $\tau_{2}$ along the axes $x_{1}$ and $x_{2}$, respectively. Under these conditions, equilibrium is guaranteed by Eq. (1).

$$
\frac{\partial \tau_{1}}{\partial x_{1}}+\frac{\partial \tau_{2}}{\partial x_{2}}=0
$$

The cohesion-less Coulomb yield function is given by Eq. (2), where $\|\cdot\|$ stands for the Euclidian norm, $\mu$ is the friction coefficient and $\sigma_{n}$ is the normal stress component. The stress component $\sigma_{n}$ is positive in tension, thus it only takes non-positive values.

$$
\|\vec{\tau}\|+\mu \sigma_{n} \leqslant 0
$$

In the plastic torsion of continuous beams, a boundary condition must be included to force the shear stress being parallel to the cross section at the edge (Nadai, 1950). Nevertheless, in the case of an interface between infinitely strong blocks, it is possible to think that there exists a narrow strip near the edge where the stress directions modify in such a way that they are parallel to the boundary at the very edge. At the limit, when the strip is narrow enough, its influence can be neglected in the calculations. This is possible due to the fact that the infinitely strong adjacent blocks can transmit the resultant stress concentrations.

The solution to the problem can be obtained by a kinematic approach. The flow rule is non-associated because the normal component is zero $\delta n=0$, but the tangential component is associated with the yield function, Eq. (2). In this way Eq. (3) provides the corresponding tangential flow. Here $\delta \gamma_{1}$ and $\delta \gamma_{2}$ are the relative displacement rates along the reference axes $x_{1}$ and $x_{2}$, respectively, and $\delta \lambda$ is the torsion flow multiplier. It is observed that the flow vector is parallel to the tangential stress vector; see also Fig. 2.

$$
\left[\begin{array}{l}
\delta \gamma_{1} \\
\delta \gamma_{2}
\end{array}\right]=\frac{1}{|\vec{\tau}| \mid}\left[\begin{array}{l}
\tau_{1} \\
\tau_{2}
\end{array}\right] \delta \lambda
$$

For a relative rotation rate in the normal direction between the two sides of the interface equal to $\delta \theta_{n}$, Eq. (4) gives the relative displacement rate of a point $P$ with coordinates $\left(x_{1}, x_{2}\right)$. The shear stress in this same point is given by Eq. (5). Here $\theta=\tan ^{-1}\left(x_{2} / x_{1}\right)$ is the angular polar coordinate and $\tau_{0}=-\mu \sigma_{n}$ is the shear 


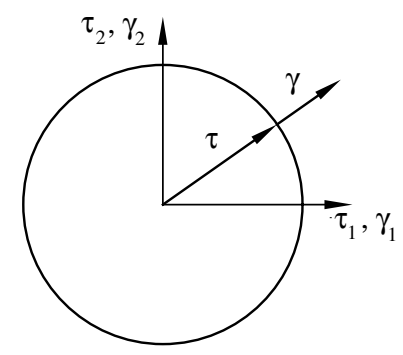

Fig. 2. Shear yield function and a flow vector.

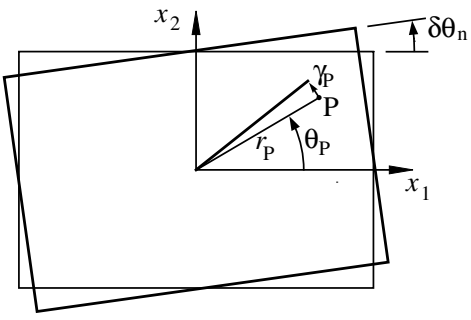

(a)

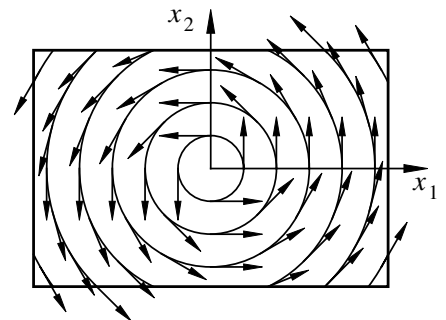

(b)

Fig. 3. Proposed torsion failure model: (a) flow vector at point $P$ and (b) stress vector field.

stress that produces sliding. The relative displacement rate and shear stress have the same direction but different magnitudes, Fig. 3.

$$
\begin{aligned}
& {\left[\begin{array}{l}
\delta \gamma_{1} \\
\delta \gamma_{2}
\end{array}\right]=\left[\begin{array}{c}
-x_{2} \\
x_{1}
\end{array}\right] \delta \theta_{n}} \\
& {\left[\begin{array}{l}
\tau_{1} \\
\tau_{2}
\end{array}\right]=\left[\begin{array}{c}
-\sin (\theta) \\
\cos (\theta)
\end{array}\right] \tau_{0}}
\end{aligned}
$$

Under the stress distribution proposed, Eq. (6) gives the torsion moment strength, $T$, of an interface. Here, $r=\sqrt{x_{1}^{2}+x_{2}^{2}}$ is the radial polar coordinate and $\mathrm{d} A=r \mathrm{~d} \theta \mathrm{d} r$ is the differential of area. If the centre of twisting (the centre of the stress distribution) is not at the centre of plastic torsion, a shear force resultant exists whose components along the axes $x_{1}$ and $x_{2}$, respectively $V_{1}$ and $V_{2}$, are given by Eqs. (7) and (8). Although the approach presented here was developed independently, in Goyal et al. (1991) an equivalent proposal can be found.

$$
\begin{aligned}
& T=\int_{\mathscr{S}} \tau_{0} r \mathrm{~d} A \\
& V_{1}=\int_{\mathscr{S}}-\tau_{0} \sin (\theta) \mathrm{d} A \\
& V_{2}=\int_{\mathscr{S}} \tau_{0} \cos (\theta) \mathrm{d} A
\end{aligned}
$$

In order to perform numerical calculations, Eqs. (6)-(8) must be integrated over the particular interface area. Nevertheless, the integrals are hard to solve analytically on shapes other than the circular one. It 


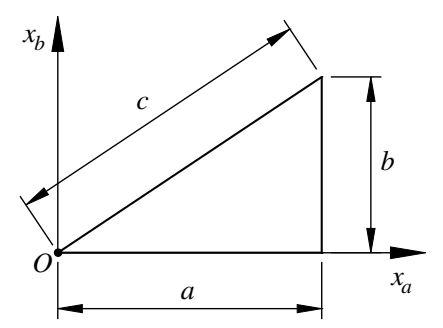

Fig. 4. Integration triangle.

was found that the best way to calculate torsion strengths under constant normal stress was to obtain the exact formulae for those equations over a right-angled triangle with a vertex on the origin, Fig. 4. Here, a new reference system is defined with axes $x_{a}$, along the adjacent to the origin leg (with length $a$ ), and $x_{b}$, parallel to the other leg (with length $b$ ). The choice of this integration triangle obeys to the fact that it is possible to find exact solutions for the above mentioned integrals and then, the torsion and shear strengths can be calculated for any polygonal section just by addition or subtraction of integrals over right-angled triangular areas. Furthermore, the centre of twisting can be placed anywhere, inside or outside the interface.

Two other integrals are useful when the shear force resultant must be calculated. These integrals represent the torsion moments, $T_{1}$ and $T_{2}$, produced independently by the $\tau_{1}$ stress, Eq. (9), and by the $\tau_{2}$ stress, Eq. (10), respectively.

$$
\begin{aligned}
& T_{1}=\int_{\mathscr{S}}-\tau_{1} x_{2} \mathrm{~d} A \\
& T_{2}=\int_{\mathscr{S}} \tau_{2} x_{1} \mathrm{~d} A
\end{aligned}
$$

With the notation of Fig. 4, the integrals over the triangular area are presented in Eqs. (11)-(15). Evidently the hypotenuse is $c=\sqrt{a^{2}+b^{2}}$, and it is straightforward to verify that $T_{1}+T_{2}=T$.

$$
\begin{aligned}
& T=\frac{-\mu \sigma_{n}}{6}\left[a b c+a^{3} \ln \left(\frac{b+c}{a}\right)\right] \\
& V_{1}=\frac{-\mu \sigma_{n}}{2}\left(a c-a^{2}\right) \\
& V_{2}=\frac{-\mu \sigma_{n}}{2}\left[a^{2} \ln \left(\frac{b+c}{a}\right)\right] \\
& T_{1}=\frac{-\mu \sigma_{n} a}{6 c}\left[b^{3}+a^{2}\left(b+c \ln \left(\frac{b+c}{a}\right)\right)\right] \\
& T_{2}=\frac{-\mu \sigma_{n}}{3}\left[a^{3} \ln \left(\frac{b+c}{a}\right)\right]
\end{aligned}
$$

No relevant experimental evidence was found in the literature about the strength of cohesion-less frictional interfaces. A comparison presented in Orduña (2003) shows that the above formulae agrees exactly with the results from simulations using finite element analysis. 


\section{Plastic torsion on rectangular interfaces}

It is possible to study the plastic torsion on rectangular interfaces with the help of the equations obtained before for right-angled triangles. In particular, it is interesting to analyse the interaction of the torsion strength with the shear forces and bending moments acting on the interface.

First, it is important to establish the torsion strength of a rectangular interface in absence of shear forces or bending moments. Fig. 5 shows a rectangular interface with the notation used, $l_{1}$ and $l_{2}$ are half the lengths of the rectangle sides parallel to the axes $x_{1}$ and $x_{2}$, respectively. In frictional interfaces it is useful to have the torsion strength in absence of shear forces or bending moments, $T_{0}$, in terms of the non-positive normal force $N$, as in Eq. (16). The torsion constant, $c_{\mathrm{T}}$, given by Eq. (17), is obtained applying Eq. (11) to the eight right-angled triangles with legs $l_{1}$ and $l_{2}$, and hypotenuse $c=\sqrt{l_{1}^{2}+l_{2}^{2}}$ in which the interface is divided, Fig. 5.

$$
\begin{aligned}
& T_{0}=c_{T} \mu(-N) \\
& c_{\mathrm{T}}=\frac{1}{3}\left[c+\frac{l_{1}^{2}}{2 l_{2}} \ln \left(\frac{l_{2}+c}{l_{1}}\right)+\frac{l_{2}^{2}}{2 l_{1}} \ln \left(\frac{l_{1}+c}{l_{2}}\right)\right]
\end{aligned}
$$

\subsection{Torsion-shear interaction}

Both shear force and torsion moment strengths of an interface are provided by shear stresses. Moving the centre of twisting away from the centroid of a rectangular interface, it is possible to obtain shear force resultants together with torsion moment. Fig. 6 shows how the shear (and flow) lines modify as the centre of twisting is moved to the left in a particular rectangular interface. As the twisting centre moves away, the torsion resultant with respect to the interface centre decreases and the shear force resultant increases. At the limit, when the twisting centre is at an infinite distance from the interface, the shear lines are straight, the torsion is zero and the shear force attains its maximum value, $V_{0}$, given by Eq. (18).

$$
V_{0}=\mu(-N)
$$

Fig. 7 presents some elements of the family of interaction curves that can be obtained for rectangular interfaces with different aspect ratios using Eqs. (11)-(15). The aspect ratio is defined as $l_{2} / l_{1}$ for a twisting centre moving as in Fig. 6. It is evident that the curves for zero and infinite aspect ratios are the lower and upper bounds for the family. The fact that pure torsion strength needs a zero shear force, and pure shear strength requires a zero torsion moment, is a consequence from the fact that both strengths depend on the available shear stress strength.

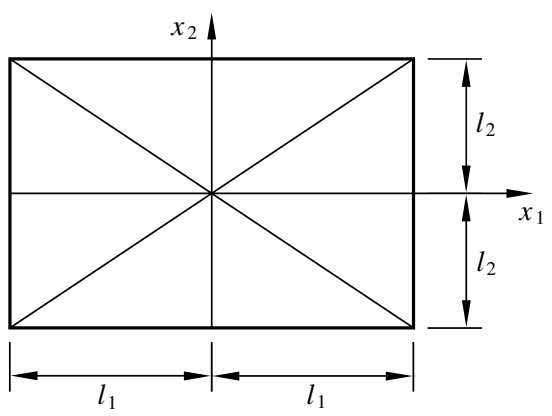

Fig. 5. Rectangular interface. 

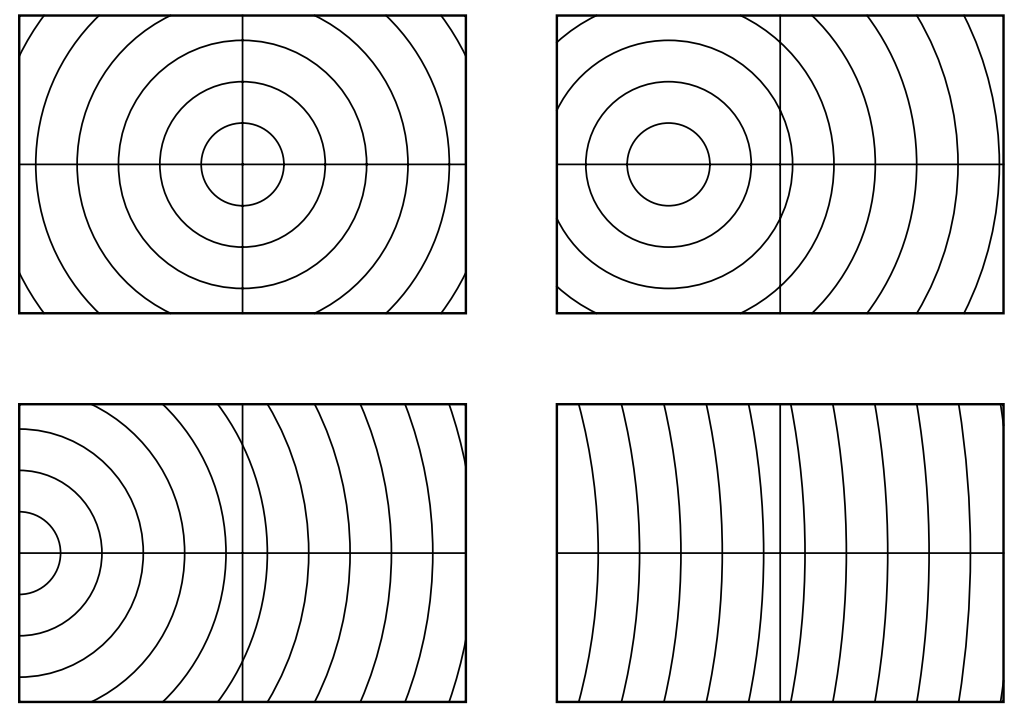

Fig. 6. Stress lines for different locations of the twisting centre.

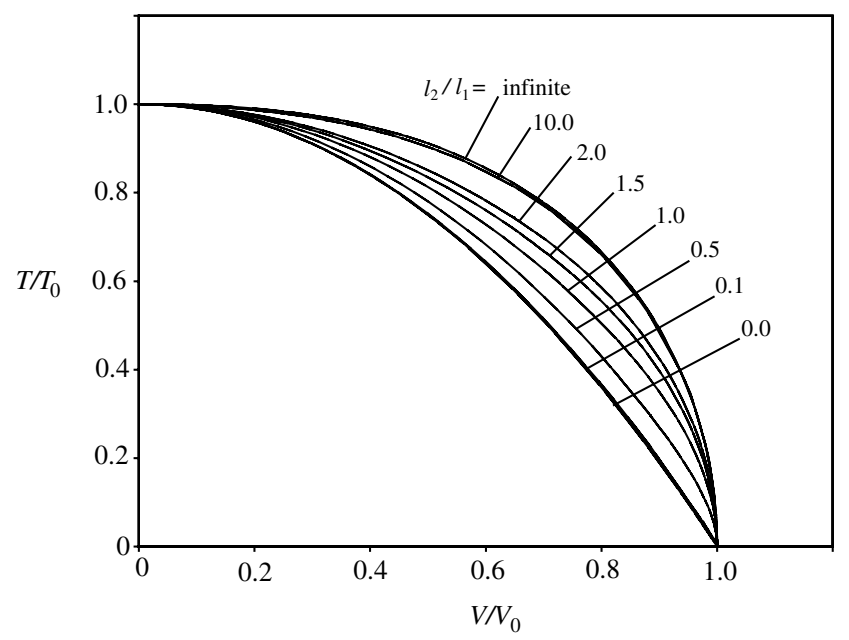

Fig. 7. Non-dimensional torsion-shear interaction for different interface aspect ratios.

Evidently, the flow for this type of stress states is a relative rotation about the twisting centre. In terms of generalised strains (or relative displacement rates) related to the interface centre, the flow consists of the relative normal rotation and relative displacements along the tangential directions. These flow components can be obtained through the associated flow condition, as the only reason of non-associativity is that the normal relative displacement component must be zero.

\subsection{Torsion-bending moment interaction}

Bending moments in the interface affect the torsion strength by modifying the normal stress distribution. If the interface is supposed to behave linearly elastic on compression and tension stresses are not allowed, 


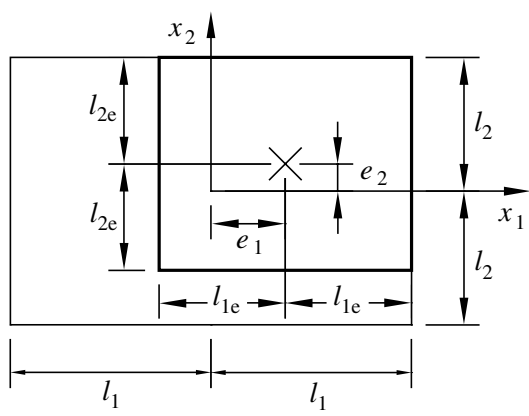

(a)

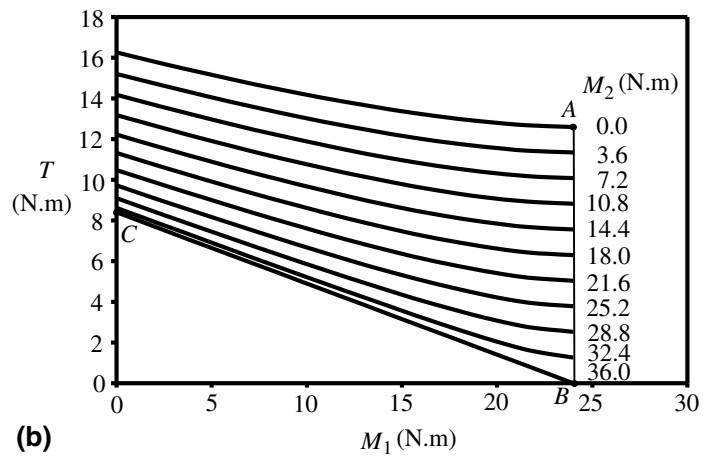

Fig. 8. Torsion-bending moment interaction: (a) effective rectangular area and (b) interaction curves for $l_{1}=0.15 \mathrm{~m}, l_{2}=0.10 \mathrm{~m}$, $\mu=0.7$ and $\sigma_{n}=-4.0 \times 10^{-3} \mathrm{~N} / \mathrm{mm}^{2}$.

when the normal force is outside the middle third, the effective area of the interface is reduced and its shape is modified. This model is rather complex to study and, in ancient masonry structures, there is no guarantee about the linear distribution of stresses in the contact area. Therefore, a simplified model is proposed instead. Hereafter, the bending moment on the interface is described by its components $M_{1}$ and $M_{2}$ along the reference axes $x_{1}$ and $x_{2}$, respectively, and follow the right hand rule.

The simplification, which seems rather obvious in design approaches, e.g. for the dimensioning of reinforced concrete structures, consists of assuming that the normal force on the interface is equilibrated by a uniform normal stress distribution on a rectangular area. Casapulla and D'Ayala (2001) used this simplification in the study of a particular limit analysis problem on arches, which involved torsion failure. The equilibrium of moments is assured by shaping the effective area in such a way that its centroid is located at the point $\left(e_{1}, e_{2}\right)$, where $e_{1}$ and $e_{2}$ are the eccentricities of the normal force along the reference axes, Fig. 8(a). The half-sides of the effective rectangle, $l_{1 e}$ and $l_{2 e}$, are given by Eqs. (19) and (20), respectively and the normal stress, $f_{c}$, is given by Eq. (21). Here it is noted again that $N$ has a negative value. Fig. 8(b) shows the interaction curves obtained for a particular case of a given geometry, friction angle and normal stress.

$$
\begin{aligned}
& l_{1 e}=l_{1}-e_{1}=l_{1}+\frac{M_{2}}{N} \\
& l_{2 e}=l_{2}-e_{2}=l_{2}-\frac{M_{1}}{N} \\
& f_{c}=\frac{N^{2}}{4\left(-l_{1} N-M_{2}\right)\left(-l_{2} N+M_{1}\right)}
\end{aligned}
$$

As shown in Fig. 8(b), the torsion strength reduces as the bending moments increase. Nevertheless, when one of the moments takes its maximum value, i.e. when a hinge forms, the torsion strength has not fallen to zero. This is easy to explain: for instance, starting with both bending moments equal to zero, and steadily increasing $M_{2}$, the effective half-side $l_{1 e}$ decreases proportionally. When $l_{1 e}$ reaches the zero value (under the infinite compression strength assumption), the effective area has been reduced to a line, a hinge forms, and the normal force is transmitted through that line. A tangential force distribution, half in the direction of $x_{1}$ and half in the opposite direction, can be associated to the previous linear normal force distribution; Fig. 9. This tangential force distribution produces a torsion moment $T_{h 2}$; Eq. (22) gives its magnitude, and point $C$ in Fig. 8(b) represents this situation graphically. Of course, on the presence of a moment in the $x_{1}$ direction, 


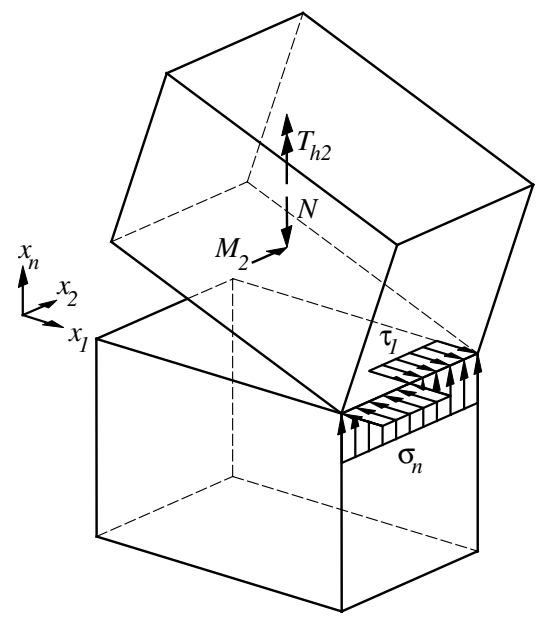

Fig. 9. Stress distribution for torsion moment $T_{h 2}$, normal force $N$ and bending moment $M_{2}$ producing a hinge.

the half-side $l_{2}$ must be replaced by its effective value $l_{2 e}$ in Eq. (22). If both moments reach their maximum values, hinges form in both directions, and the effective area reduces to a contact point, in this case the torsion strength is zero as the model correctly reflects; point $B$ in Fig. 8(b). It is observed that the vertical line, from point $A$ to point $B$ in Fig. 8(b), and the lower line, from point $C$ to point $B$ in the same graph, represent the intersections with the hinging criteria. In the case of using limited compressive stresses, the hinge yield conditions will automatically limit the minimum size of the effective area and, therefore, will move the line $A-B$ to the left and the line $C-B$ upwards.

$$
T_{h 2}=-\frac{1}{2} \mu N l_{2}
$$

The flow corresponding to this yield mode consists only of a relative rotation in the normal direction about the centroid of the effective area. The only role of the bending moment is to reduce the effective interface area, and otherwise, pure torsion failure continues to be assumed so, no bending rotations occur unless a hinging criterion is also active. Related to the centre of the interface, the flow will consist, apart of this normal rotation, of relative displacements in the tangential directions. Observe that the yield function, although not explicitly stated here, depends on the torsion moment, normal force and bending moments, but no flow components exist in the direction of the bending rotations. Besides, the flow rule has components in the tangential displacement rates but the yield function does not depend on the shear forces. This is another source of non-associativity of the flow rule for this yield mode.

The study of torsion strength considering interactions with both bending moments and shear forces seems straightforward. Once determined the effective interface area and the availability of shear stress strength through the bending moments and normal force, the torsion-shear interaction is treated on this effective area. Experimental validation of the proposed yield surface is outside the scope of the present paper, as no test results are available from the literature. Nevertheless, it is stressed that: (a) the simplifications seem reasonable; (b) several failure surfaces used for non-linear material models, used in the literature for simulations, provide a solution close to experimental results only in few selected load-paths (e.g. uniaxial tension or compression), being comprehensive experimental data absent for the full multi-stress envelop. This is similar to the process followed by the authors; (c) there is no experimental evidence to assess the validity of the proposed simplifications at the failure surface level and (d) validation of the assumptions at structural level is provided from the analysis carried out in Orduña and Lourenço (2005). 


\section{Proposed yield surface}

Mathematical programming routines work better as the expressions used for defining the problem are simpler. In this section, a simplified yield function is proposed. The proposal includes a piecewise linear approximation to the torsion yield function for rectangular interfaces outlined above, which includes the pure shear failure. The yield function is complemented with a hinging failure model for quadrilateral interfaces capable to take into account limited compressive stresses. The interactions of torsion strength with shear forces and bending moments are considered independently. As mentioned above, the combined interaction was not studied due to the lack of experimental evidence to validate the model.

In order to work already within the three-dimensional limit analysis framework, it is convenient to make the following definitions. Two reference system types exist: the global reference system with coordinates $(X, Y, Z)$, and the local reference system associated to each interface. The local system for the interface $k$, which is the interface between the blocks $i$ and $j$, is defined by the unitary vectors $\vec{s}_{1 k}, \vec{s}_{2 k}$, and $\vec{n}_{k}$ along the $x_{1}, x_{2}$ and $x_{n}$ axes of that particular interface. These vectors are normal to each other and form a righthanded system, such that $\vec{n}_{k}$ is normal to the interface and points to the outside of block $i$, as in Fig. 10. The matrix $\vec{T}_{k}^{g}$ transforms a vector in the global system to the interface $k$ local system.
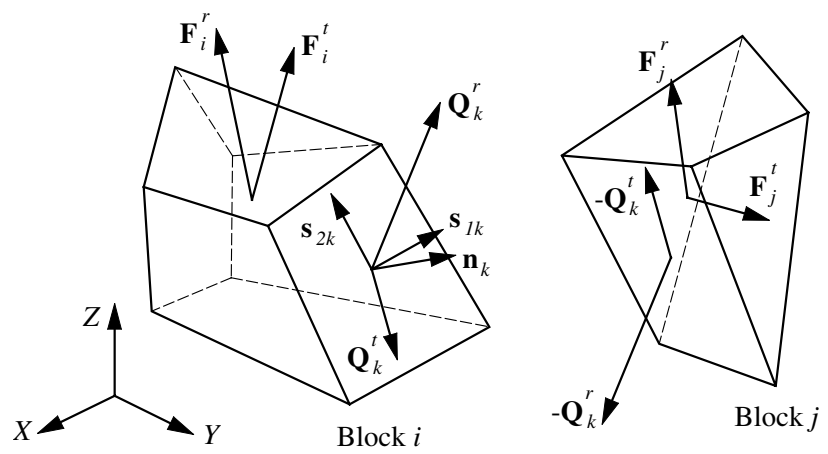

Fig. 10. Static variables at an interface and adjacent blocks.

Table 1

Interface $k$ variable vectors

\begin{tabular}{lll}
\hline Vector & Components & Description \\
\hline$\vec{Q}_{k}^{t}$ & $V_{1 k}$ & Shear force along $\vec{s}_{1 k}$ \\
& $V_{2 k}$ & Shear force along $\vec{s}_{2 k}$ \\
& $N_{k}$ & Normal force along $\vec{n}_{k}$ \\
$\vec{Q}_{k}^{r}$ & $M_{1 k}$ & Bending moment along $\vec{s}_{1 k}$ \\
& $M_{2 k}$ & Bending moment along $\vec{s}_{2 k}$ \\
& $T_{k}$ & Torsion moment along $\vec{n}_{k}$ \\
$\delta \vec{q}_{k}^{t}$ & $\delta s_{1 k}$ & Relative translation displacement rate along $\vec{s}_{1 k}$ \\
& $\delta s_{2 k}$ & Relative translation displacement rate along $\vec{s}_{2 k}$ \\
& $\delta n_{k}$ & Relative translation displacement rate along $\vec{n}_{k}$ \\
& $\delta \theta_{1 k}$ & Relative angular displacement rate along $\vec{s}_{1 k}$ \\
& $\delta \theta_{2 k}$ & Relative angular displacement rate along $\vec{s}_{2 k}$ \\
\hline
\end{tabular}


Table 2

Block $i$ variable vectors

\begin{tabular}{lll}
\hline Vector & Components & Description \\
\hline$\delta \vec{u}_{i}^{t}$ & $\delta u_{i}$ & Translation displacement rate in $X$ direction \\
& $\delta v_{i}$ & Translation displacement rate in $Y$ direction \\
& $\delta w_{i}$ & Translation displacement rate in $Z$ direction \\
$\vec{u}_{i}^{r}$ & $\delta \omega_{X i}$ & Angular displacement rate in $X$ direction \\
& $\delta \omega_{Y i}$ & Angular displacement rate in $Y$ direction \\
& $\delta \omega_{Z i}$ & Angular displacement rate in $Z$ direction \\
$\vec{F}_{i}^{t}$ & $f_{X i}$ & Force in $X$ direction \\
& $f_{Y i}$ & Force in $Y$ direction \\
$\vec{F}_{i}^{r}$ & $f_{Z i}$ & Force in $Z$ direction \\
& $m_{X i}$ & Moment in $X$ direction \\
& $m_{Y i}$ & Moment in $Y$ direction \\
\hline
\end{tabular}

The static variables, or generalised stresses at an interface $k$, are split into the force vector $\vec{Q}_{k}^{t}$ and the moment vector $\vec{Q}_{k}^{r}$, both in local coordinates and being the force vector applied at the interface centroid. Table 1 contains the description of the components of the static variables vectors. The vector $\vec{Q}$ gathers the generalised stresses for all the interfaces in the model. Correspondingly, the kinematic variables, or generalised strains for the same interface $k$ are the relative translation displacement rates $\delta \vec{q}_{k}^{t}$ and the relative angular displacement rates $\delta \vec{q}_{k}^{r}$. Table 1 also describes the components of these vectors. Again, the vector $\delta \vec{q}$ gathers the generalised strains for all the interfaces in the model.

The degrees of freedom for the generic block $i$ are the displacement rates at the block centroid $\delta \vec{u}_{i}^{t}$ and the angular displacement rates $\delta \vec{u}_{i}^{r}$. These vectors are referred to the global system and their description is given in Table 2 . The vector $\delta \vec{u}$ gathers the displacement rates for all the blocks in the model. The loads applied to the block $i$ are the force vector $\vec{F}_{i}^{t}$ at the block centroid, and the moment vector $\vec{F}_{i}^{r}$, see also Table 2 and Fig. 10. These load vectors have a constant part $\vec{F}_{i c}^{t}, \vec{F}_{i c}^{r}$ and a variable part $\vec{F}_{i v}^{t}, \vec{F}_{i v}^{r}$. The vectors $\vec{F}_{c}$ and $\vec{F}_{v}$ gather the constant and variable loads over all the blocks in the model, respectively.

Once defined the notation, the piecewise linear approximation to the torsion yield function is presented in the following subsections.

\subsection{Pure torsion}

The first part of the piecewise linear approximation for the torsion yield function corresponds to the pure torsion condition. The maximum torsion moment is upper and lower bounded by the Eq. (23). This follows directly from Eq. (16).

$$
\varphi_{\mathrm{twist} 1,2} \equiv \frac{|T|}{c_{\mathrm{T}}}+\mu N \leqslant 0
$$

\subsection{Torsion-shear interaction}

Fig. 11(a) presents the bounding curves of the torsion-shear interaction corresponding to interface aspect ratios of zero and infinity addressed above. The broken line in the graph represents the proposed approximation. This approximated function does not take into account the interface aspect ratio, firstly because its influence is not so large, and secondly, because its consideration would unnecessarily complicate 

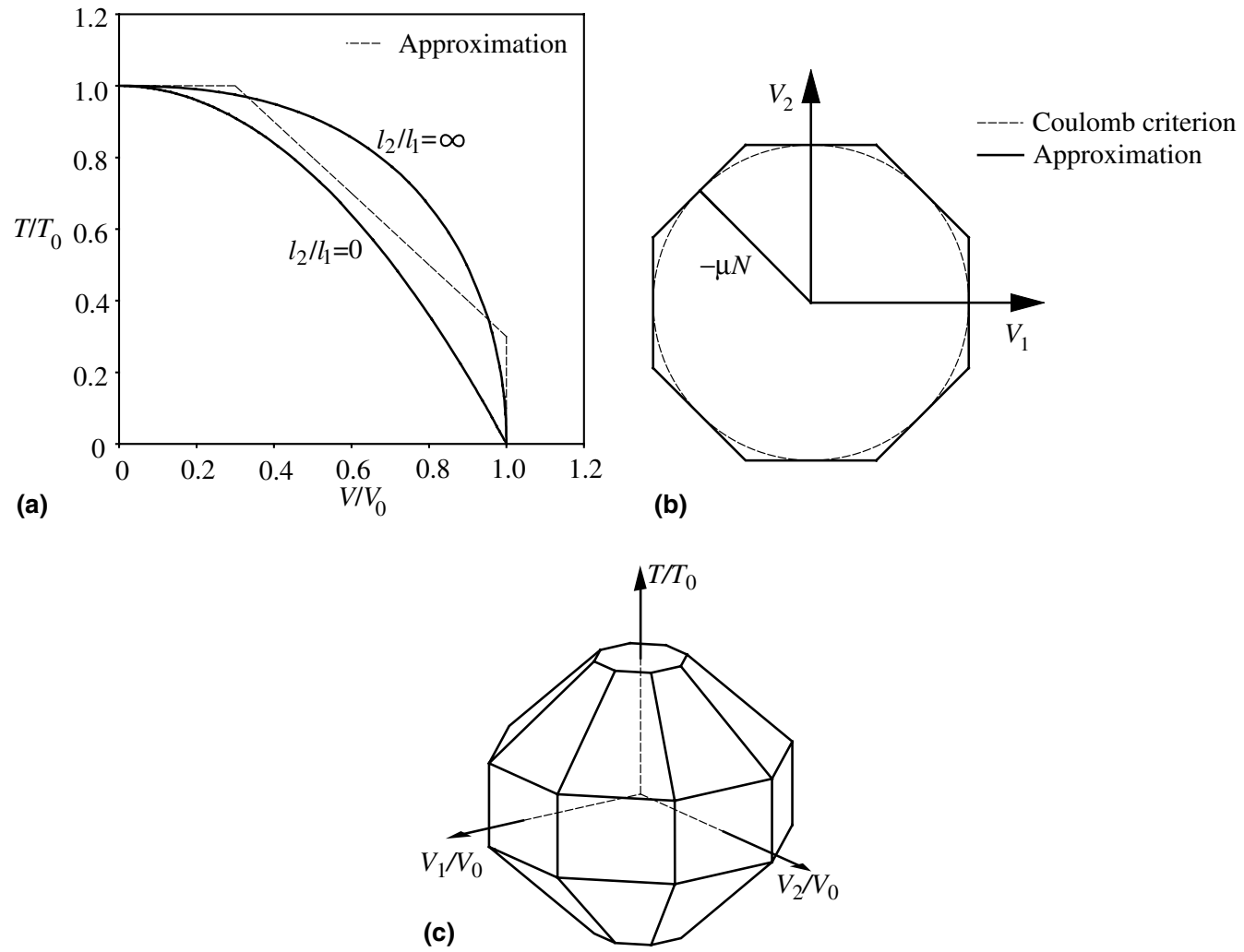

Fig. 11. Non-dimensional torsion-shear interaction: (a) upper and lower interaction curves and approximation; (b) pure shear condition and (c) three-dimensional yield surface representation.

the expressions. The complete yield function is symmetric with respect to the $V / V_{0}$ axis. This axis represents, in principle, any shear direction because the approximated yield function would be axisymmetric with respect to the $T / T_{0}$ axis. Nevertheless, also the pure shear criterion is linearly approximated by eight functions. Fig. 11(b) shows the cohesion-less Coulomb criterion in doted line and the proposed approximation in continuous line. The horizontal branch in Fig. 11(a) represents Eq. (23). The vertical branch is the pure shear criterion, represented by the eight planes of Eqs. (24)-(26), also represented in Fig. 11(b). The inclined branch corresponds approximately to the side of an octagon inscribed in a unitary radius circle. The mathematical representation of this branch in the four quadrants of the $T-V_{1}$ plane conducts to Eq. (27). Those corresponding to the $T-V_{2}$ plane are in Eq. (28). Finally, there are eight more functions, Eq. (29), for planes at $45^{\circ}$ from the $V_{1}$ and $V_{2}$ axes. Fig. 11(c) is a three-dimensional representation of the set of proposed yield functions.

$$
\begin{aligned}
& \varphi_{\text {slip } 1,2} \equiv\left|V_{1}\right|+\mu N \leqslant 0 \\
& \varphi_{\text {slip } 3,4} \equiv\left|V_{2}\right|+\mu N \leqslant 0 \\
& \varphi_{\text {slip } 5-8} \equiv \frac{\left|V_{1}\right|+\left|V_{2}\right|}{\sqrt{2}}+\mu N \leqslant 0
\end{aligned}
$$


Table 3

Flow directions for torsion-shear interaction

\begin{tabular}{lllllll}
\hline Flow multiplier & $\delta s_{1}$ & $\delta s_{2}$ & $\delta n$ & $\delta \theta_{1}$ & $\delta \theta_{2}$ & $\delta \theta_{n}$ \\
\hline$\delta \lambda_{\text {slip } 1,2}$ & $\frac{V_{1}}{\left|V_{1}\right|}$ & 0 & 0 & 0 & 0 & 0 \\
$\delta \lambda_{\text {slip3,4 }}$ & 0 & $\frac{V_{2}}{\left|V_{2}\right|}$ & 0 & 0 & 0 & 0 \\
$\delta \lambda_{\text {slip5-8 }}$ & $\frac{V_{1}}{\left|V_{1}\right| \sqrt{2}}$ & $\frac{V_{2}}{\left|V_{2}\right| \sqrt{2}}$ & 0 & 0 & 0 & 0 \\
$\delta \lambda_{V_{1} T 1-4}$ & $\frac{V_{1}}{\left|V_{1}\right|}$ & 0 & 0 & 0 & 0 & $\frac{T}{|T| c_{\mathrm{T}}}$ \\
$\delta \lambda_{V_{2} T 1-4}$ & 0 & $\frac{V_{2}}{\left|V_{2}\right|}$ & 0 & 0 & 0 & $T$ \\
$\delta \lambda_{V_{12} T 1-8}$ & $\frac{V_{1}}{\left|V_{1}\right| \sqrt{2}}$ & $\frac{V_{2}}{\left|V_{2}\right| \sqrt{2}}$ & 0 & 0 & 0 & $T$ \\
\hline
\end{tabular}

$$
\begin{aligned}
\varphi_{V_{1} T 1-4} & \equiv \frac{|T|}{c_{\mathrm{T}}}+\left|V_{1}\right|+1.3 \mu N \leqslant 0 \\
\varphi_{V_{2} T 1-4} & \equiv \frac{|T|}{c_{\mathrm{T}}}+\left|V_{2}\right|+1.3 \mu N \leqslant 0 \\
\varphi_{V_{12} T 1-8} & \equiv \frac{|T|}{c_{\mathrm{T}}}+\frac{\left|V_{1}\right|+\left|V_{2}\right|}{\sqrt{2}}+1.3 \mu N \leqslant 0
\end{aligned}
$$

The rows of Table 3 have the flow directions for the flow multipliers corresponding to the yield functions in Eqs. (24)-(29). All these directions have a zero normal component, but otherwise are obtained by the normality condition.

\subsection{Torsion-bending moment interaction}

The approximation chosen for the torsion-bending moment interaction is a series of planes. Fig. 12 presents a graph with the interaction curves for three values of $M_{2}$ on the same interface as Fig. 8(b). The approximation plane on the first octant of the $M_{1}-M_{2}-T$ space contains the points $A, B$ and $C$ of these graphs. This plane overestimates in general the torsion strength. Only on the straight lines $A-B$ and $C-$ $B$ the approximation coincides with the effective area model. The pure torsion condition, Eq. (23), limits the maximum value of the torsion moment. This condition is represented in Fig. 12 by the horizontal line at $T=16.3 \mathrm{~N} \mathrm{~m}$. The lines $A-B$ and $C-B$ correspond to the intersection of the torsion-moment surface with the hinging surfaces and it has been observed that very often the stresses lie on these lines at failure. In fact,

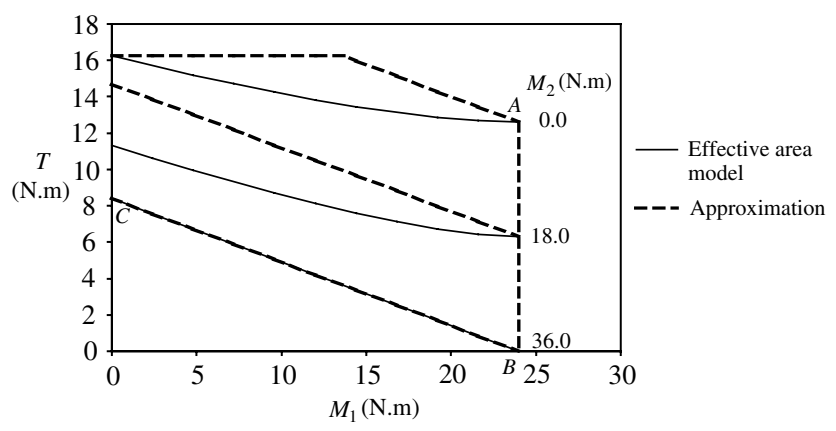

Fig. 12. Graph of torsion-bending moment interaction on a rectangular interface for $l_{1}=0.15 \mathrm{~m}, l_{2}=0.10 \mathrm{~m}, \mu=0.7$ and $\sigma_{n}=-4.0 \times 10^{-3} \mathrm{~N} / \mathrm{mm}^{2}$. 
Table 4

Key stress states for torsion-bending moment interaction

\begin{tabular}{llll}
\hline & $M_{1}$ & $M_{2}$ & $T$ \\
\hline Point $A$ & $-l_{2} N$ & 0 & $-\frac{1}{2} \mu N l_{1}$ \\
Point $B$ & $-l_{2} N$ & $-l_{1} N$ & 0 \\
Point $C$ & 0 & $-l_{1} N$ & $-\frac{1}{2} \mu N l_{2}$ \\
\hline
\end{tabular}

it is a well-known aspect of plastic analysis dealing with multi-surface yield functions that the intersections of the yield surfaces "attract" the stress states. Therefore, even if the approximated model can overestimate the torsion moment strength, it is considered appropriate in general.

Points $A, B$ and $C$ in Fig. 8(b) have the stress states given in Table 4. Eq. (30) is the yield function corresponding to the plane defined by these three points, as well as for the planes in the remaining octants of the $M_{1}-M_{2}-T$ space. In the bending moments directions, the stress space is limited by the hinging yield functions. Finally, the torsion moment is also limited by Eq. (23), as already mentioned. Fig. 13 is a three-dimensional representation of the complete yield function for the interface considered. The points $A, B$ and $C$ are identified for reference.

$$
\varphi_{M_{12} T 1-8} \equiv \frac{|T|}{l_{1}+l_{2}}+\frac{\mu}{2}\left(\frac{\left|M_{1}\right|+\left|M_{2}\right|}{l_{1}+l_{2}}+N\right) \leqslant 0
$$

The flow directions consist of a rotation about the $\left(e_{1}, e_{2}\right)$ point with the same direction as the torsion moment; Fig. 14. As the generalised strains are referred to the interface centroid, tangential relative displacements accompany the rotation. The first and second rows of Table 5 present the flow directions for positive and negative torsion moments, respectively. Here $\varepsilon$ is a small positive number introduced in order to avoid divisions by zero when $N=0$, and remember that $e_{1}=-M_{2} / N$ and $e_{2}=M_{1} / N$.

In practice, it is useful to dispose of an interface model for more general shapes than rectangular ones. Hereafter, a quadrilateral shape is considered. Fig. 15 shows the naming convention used for the vertices and sides of a generic quadrilateral interface. In order to use the previous model for an interface of this shape, it is transformed into an approximated rectangular shape with mid-sides $l_{1}$ and $l_{2}$ given by Eqs. (31) and (32).

$$
\begin{aligned}
& l_{1}=\frac{1}{4}\left(x_{1}^{1}+x_{1}^{2}-x_{1}^{3}-x_{1}^{4}\right) \\
& l_{2}=\frac{1}{4}\left(x_{2}^{2}+x_{2}^{3}-x_{2}^{1}-x_{2}^{4}\right)
\end{aligned}
$$

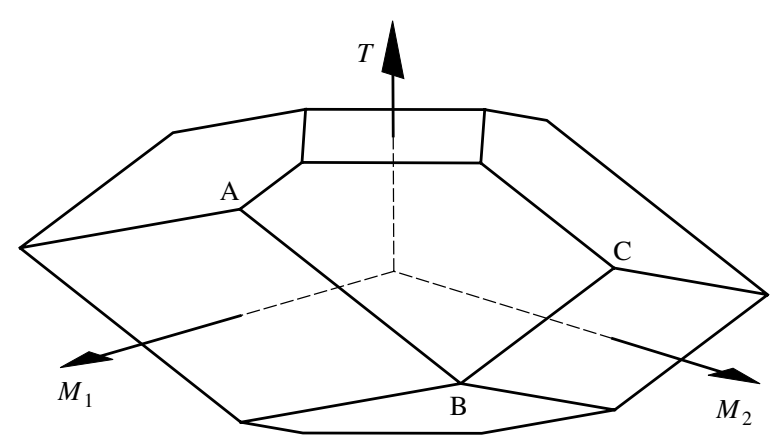

Fig. 13. Torsion-bending moment interaction. 


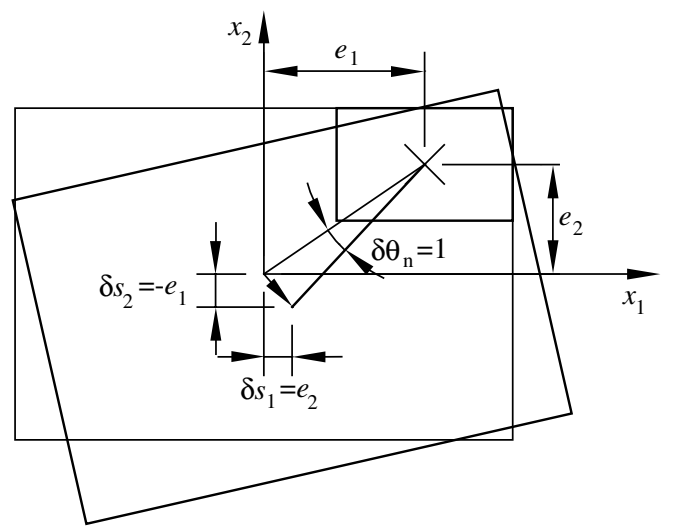

Fig. 14. Flow due to twisting with non-zero bending moments.

Table 5

Flow directions for torsion-bending moment interaction

\begin{tabular}{lllllll}
\hline & $\delta s_{1}$ & $\delta s_{2}$ & $\delta n$ & $\delta \theta_{1}$ & $\delta \theta_{2}$ & $\delta \theta_{n}$ \\
\hline$\delta \lambda_{M_{12} T 1-4}$ & $M_{1} /(N-\varepsilon)$ & $M_{2} /(N-\varepsilon)$ & 0 & 0 & 0 & 1 \\
$\delta \lambda_{M_{12} T 5-8}$ & $-M_{1} /(N-\varepsilon)$ & $-M_{2} /(N-\varepsilon)$ & 0 & 0 & 0 & -1 \\
\hline
\end{tabular}

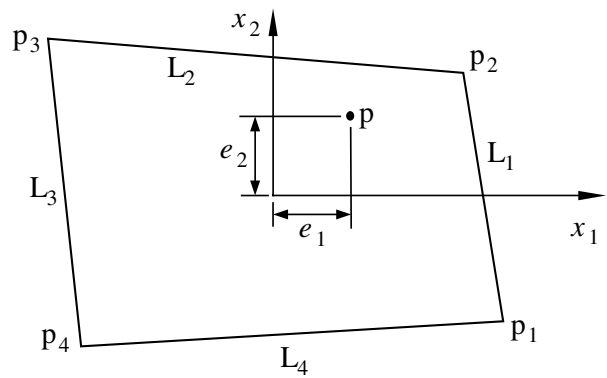

Fig. 15. Interface naming convention.

\subsection{Hinging}

Under the infinite compressive strength hypothesis, if the normal stress resultant is inside the interface, then it is safe against hinging. Consider the side $L_{1}$ in Fig. 15 and consider that the resultant of the normal stresses is applied at the point $p$, with local coordinates given by the eccentricities $\left(e_{1}, e_{2}\right)$. If this point is inside the interface (to the left of $L_{1}$ ), the normal component of the vector $\vec{h}_{1}$, defined in Eq. (33), must be grater than zero. Here $\vec{p}_{1}, \vec{p}_{2}$ and $\vec{p}$ are the position vectors of the points $p_{1}, p_{2}$ and $p$, respectively. The previous condition is expressed by Eq. (34), where the superscripts refer to the interface vertex. Taking into account that $e_{1}=-M_{2} / N$ and $e_{2}=M_{1} / N$ it is possible to obtain Eq. (35), in terms of the generalised stresses. 


$$
\begin{aligned}
& \vec{h}_{1} \equiv\left(\vec{p}_{2}-\vec{p}_{1}\right) \wedge\left(\vec{p}-\vec{p}_{1}\right) \\
& e_{1}\left(\frac{x_{2}^{1}-x_{2}^{2}}{x_{1}^{1} x_{2}^{2}-x_{1}^{2} x_{2}^{1}}\right)+e_{2}\left(\frac{x_{1}^{2}-x_{1}^{1}}{x_{1}^{1} x_{2}^{2}-x_{1}^{2} x_{2}^{1}}\right)+1 \geqslant 0 \\
& M_{1}\left(\frac{x_{1}^{2}-x_{1}^{1}}{x_{1}^{1} x_{2}^{2}-x_{1}^{2} x_{2}^{1}}\right)+M_{2}\left(\frac{x_{2}^{2}-x_{2}^{1}}{x_{1}^{1} x_{2}^{2}-x_{1}^{2} x_{2}^{1}}\right)+N \leqslant 0
\end{aligned}
$$

Other three similar equations must be defined for the remaining sides of the interface. Eq. (36) is the generic expression of the four hinging yield functions. The constants $c_{1}$ and $c_{2}$ are defined in Eq. (37), where the node coordinates index obeys a cyclic convention such that for $i=4$, then $i+1=1$ holds.

$$
\begin{aligned}
& \varphi_{\text {hinge } i} \equiv c_{1}^{i} M_{1}+c_{2}^{i} M_{2}+N \leqslant 0 \\
& c_{1}^{i}=\frac{x_{1}^{i+1}-x_{1}^{i}}{x_{1}^{i} x_{2}^{i+1}-x_{1}^{i+1} x_{2}^{i}} \\
& c_{2}^{i}=\frac{x_{2}^{i+1}-x_{2}^{i}}{x_{1}^{i} x_{2}^{i+1}-x_{1}^{i+1} x_{2}^{i}}
\end{aligned}
$$

In order to include the limit to the compressive stress in an approximate but simplified way, an additional quadratic term on $N$ can be included, similarly to the two-dimensional case (Orduña and Lourenço, 2003). This term reduces to zero the moment strength provided by the normal force when the normal stress reaches its limit value. In such a way, Eq. (39) expresses the yield functions for limited compressive stresses, and Eq. (40) provides the value of the constant $c_{3}$. Here, $A_{r}$ is the interface area and $f_{c e f}$ is the effective compressive stress. It is observed that this approach can be generalised to polygonal interfaces with any number of sides provided they are convex. Table 6 presents the associate flow components corresponding to the hinging yield mode.

$$
\begin{aligned}
& \varphi_{\text {hinge } i} \equiv c_{1}^{i} M_{1}+c_{2}^{i} M_{2}+N+c_{3} N^{2} \leqslant 0 \\
& c_{3}=\frac{1}{A_{r} f_{c e f}}
\end{aligned}
$$

The effective stress value $f_{c e f}$ is calculated by means of Eq. (41). This expression has been borrowed from reinforced concrete limit analysis theory (Nielsen, 1999). Here, $f_{c}$ is the uniaxial compressive strength of the material, and $v_{e}$ is an effectiveness factor that takes into account reductions in the compressive strength due to transverse cracking, as well as to the fact that limit analysis assumes a rigid-plastic behaviour, while, in fact, softening occurs. Eq. (42) is an expression for the effectiveness factor commonly used for concrete (Nielsen, 1999), where $f_{c}$ is expressed in $\mathrm{N} / \mathrm{mm}^{2}$.

$$
\begin{aligned}
& f_{c e f}=v_{e} f_{c} \\
& v_{e}=0.7-\frac{f_{c}}{200}
\end{aligned}
$$

Table 6

Hinging flow direction

\begin{tabular}{lllllll}
\hline Flow multiplier & $\delta s_{1}$ & $\delta s_{2}$ & $\delta n$ & $\delta \theta_{1}$ & $\delta \theta_{2}$ & $\delta \theta_{n}$ \\
\hline$\delta \lambda_{\text {hinge } i}$ & 0 & 0 & $1+2 c_{3} N$ & $c_{1}^{i}$ & $c_{2}^{i}$ & 0 \\
\hline
\end{tabular}




\section{Limit analysis formulation of three-dimensional rigid block assemblages}

In the present section, the limit analysis formulation for three-dimensional rigid blocks assemblages is presented. It is noted that this formulation assumes that full contact exists at all interfaces before the load application. In mortared masonries, this assumption can be regarded as appropriate because, at least at construction time, the mortar allowed for full contact between masonry pieces. In dry jointed masonries, or if mortar is highly deteriorated, the full contact assumption is more questionable. Since in these cases the real contact conditions are difficult to asses, the full contact hypothesis can be regarded as a statistically mean condition, i.e. if the contact consists on a number of points, lines or limited area contacts, the mean condition is that these contact issues are uniformly distributed within the interface. Therefore, it seems that the full contact assumption is appropriate in general, particularly when nonassociated flow is adopted, which implies that block interlocking upon sliding does not occur. It is noted that experiments on dry jointed shear walls (Oliveira, 2003; Vasconcelos and Lourenço, 2004) show that poor construction tolerances, which in turn affect the initial contact conditions, produce larger variability of the results than tighter tolerances. Therefore, the initial contact conditions affect the overall behaviour of the structure. This issue can not be taken into account by direct solution methods, but can be included in the load-path following solution procedure proposed by Orduña and Lourenço (2005).

The interface and block variables were defined in Section 4. Generally, for limit analysis and non-linear analysis, a load factor is defined as the ratio of the applied load with respect to a reference set of loads. Here, the load factor, $\alpha$, is the amount of the variable load vector applied to the structure. The ultimate load factor is the value of the load factor that the analyst wants to determine for safety and analysis purposes, and is associated with the collapse mechanism obtained for the structure. The total load vector, $\vec{F}$, is given by Eq. (43).

$$
\vec{F}=\vec{F}_{c}+\alpha \vec{F}_{v}
$$

\subsection{Compatibility}

The compatibility between the interface $k$ generalised strains and the displacement rates of the adjacent blocks $i$ and $j$ is guaranteed by Eq. (44). Here $\vec{c}_{k}$ is the position vector of the interface $k$ centroid, while $\vec{c}_{i}$ and $\vec{c}_{j}$ are the position vectors of blocks $i$ and $j$ centroids, respectively. Further manipulation of this equation gives Eq. (45), where the elemental compatibility matrix $\vec{C}_{k / j}$ is given in Eq. (46). Here again, $(X, Y, Z)$ refer to the global reference system and indicate the centroid coordinates of each block, $i$ and $j$, and interface, $k$, according to the subscripts. The assemblage of the equations for all the interfaces in the structure conducts to Eq. (47), where $\vec{C}$ is, evidently, the compatibility matrix of the model.

$$
\begin{aligned}
& {\left[\begin{array}{l}
\delta \vec{q}_{k}^{t} \\
\delta \vec{q}_{k}^{r}
\end{array}\right]=\left[\begin{array}{c}
\vec{T}_{k}^{g}\left(\delta \vec{u}_{j}^{t}-\delta \vec{u}_{i}^{t}+\delta \vec{u}_{j}^{r} \wedge\left(\vec{c}_{k}-\vec{c}_{j}\right)-\delta \vec{u}_{i}^{r} \wedge\left(\vec{c}_{k}-\vec{c}_{i}\right)\right) \\
\vec{T}_{k}^{g}\left(\delta \vec{u}_{j}^{r}-\delta \vec{u}_{i}^{r}\right)
\end{array}\right]} \\
& \delta \vec{q}_{k}=\vec{C}_{k / j} \delta \vec{u}_{j}-\vec{C}_{k / i} \delta \vec{u}_{i}
\end{aligned}
$$




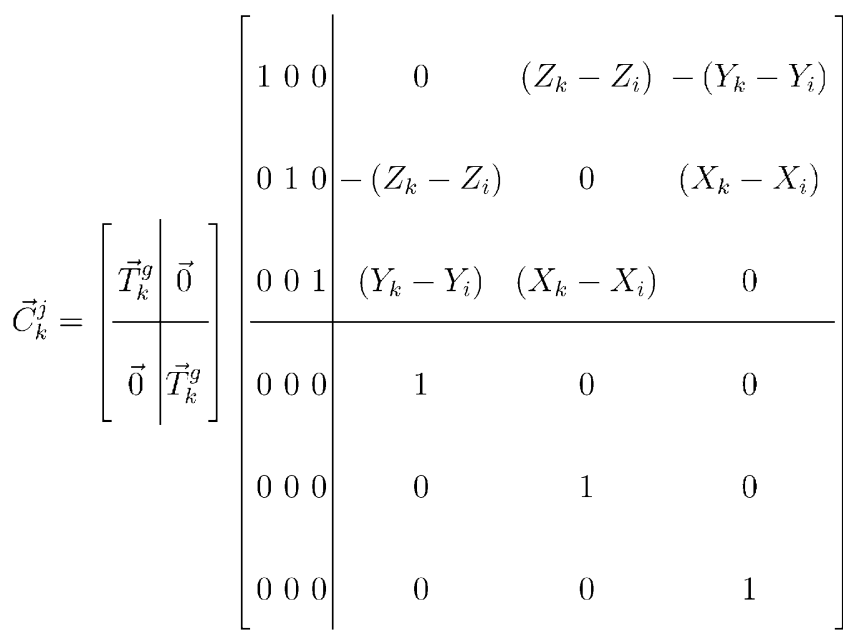

$\delta \vec{q}=\vec{C} \delta \vec{u}$

\subsection{Equilibrium} 1977).

Applying the contragredience principle, the equilibrium requirement is expressed by Eq. (48) (Grierson,

$$
\vec{F}=\vec{C}^{T} \vec{Q}
$$

\subsection{Yield function}

All the yield functions for the generic interface $k$ are gathered in the vector $\vec{\varphi}_{k}$. For a rigid blocks model, the vector $\vec{\varphi}$ contains the yield functions for all the interfaces. In this way, the yield condition for the whole structure is expressed by Eq. (49).

$$
\vec{\varphi} \leqslant \overrightarrow{0}
$$

\subsection{Flow rule}

The generalised strains for the interface $k$ calculated by the flow rule are given by Eq. (50). The columns of the matrix $\vec{N}_{0 k}$ are the flow directions for each yield mode in the interface, addressed in Section 4 . The vector $\delta \vec{\lambda}_{k}$ contains the flow multipliers corresponding to each yield function in the interface. For the whole structure the flow rule can be written as Eq. (51). Here the matrix $\vec{N}_{0}$ and the vectors $\delta \vec{q}$ and $\delta \vec{\lambda}$ are assembled for all the interfaces in the structure.

$$
\begin{gathered}
\delta \vec{q}_{k}=\vec{N}_{0 k} \delta \vec{\lambda}_{k} \\
\delta \vec{q}=\vec{N}_{0} \delta \vec{\lambda}
\end{gathered}
$$

Two additional conditions must be included in order to completely describe the plastic behaviour in mathematical terms. The first one refers to the fact that flow must involve energy dissipation i.e. the flow multipliers must be non-negative, Eq. (52). Secondly, flow can not occur unless the stresses have reached the 
yield function. Eq. (53) guarantees this, and is usually referred as consistency condition. Besides, in the mathematical programming literature, this expression is referred as complementarity condition.

$$
\begin{aligned}
& \delta \vec{\lambda} \geqslant \overrightarrow{0} \\
& \vec{\varphi}^{T} \cdot \delta \vec{\lambda}=0
\end{aligned}
$$

\subsection{The mathematical problem}

Eqs. (54)-(59) are the conditions that a limit analysis solution with non-associated flow rule must fulfil. Eq. (54) combines the compatibility and flow rule conditions. Eq. (55) is a scaling condition for the displacement rates that ensures the existence of non-zero and finite values. Eq. (56) is the equilibrium condition. Eq. (57) guaranties that the yield functions are not violated and Eq. (58) ensures that plastic flow implies energy dissipation. Finally, Eq. (59) guaranties that plastic flow cannot occur unless the stresses have reached the yield surface.

$$
\begin{aligned}
& \vec{N}_{0} \delta \vec{\lambda}-\vec{C} \delta \vec{u}=\overrightarrow{0} \\
& \vec{F}_{v}^{T} \cdot \delta \vec{u}-1=0 \\
& \vec{F}_{c}+\alpha \vec{F}_{v}-\vec{C}^{T} \vec{Q}=\overrightarrow{0} \\
& \vec{\varphi} \leqslant \overrightarrow{0} \\
& \delta \vec{\lambda} \geqslant \overrightarrow{0} \\
& \vec{\varphi}^{T} \cdot \delta \vec{\lambda}=0
\end{aligned}
$$

This set of equations corresponds to the mixed limit analysis formulation (Kamenjarzh, 1996) and represents a case known in the mathematical programming literature as a Mixed Complementarity Problem (MCP) (Ferris and Tin-Loi, 2001). In general, there is no unique solution for this problem in the presence of non-associated flow rules. If the load factor is minimised, as proposed by Baggio and Trovalusci (1998), the solution can severely underestimate the ultimate load factor, as shown in Orduña and Lourenço (2005). This affirmation refers to the fact that, minimising the load factor conducts, in some cases, to a significantly lower value of the ultimate load factor than the one computed by non-linear finite element analyses with linear-elastic blocks and elastic-perfectly plastic interfaces. It seems that the lack of taking into account the loading history in the limit analysis approach (included for this reason in the direct methods class) can have a significant impact on the result in the presence of non-associated flow rules. The safety assessment of structures is generally based on the maximum reliable strength that the structure can develop, regardless after the peak load, the strength could reduce to a residual value. Therefore, it is important to know, not only the solution with the minimum load factor, but whether this solution is reachable or not from the set of stress states that are possible under permanent loads only and satisfying the equilibrium and yield conditions, through a continuous stress path with the same requirements, and without having to apply larger load factors than the ultimate load factor. In fact, it would be possible to obtain all the solutions to the MCP, Eqs. (54)-(59), with a procedure similar to that proposed by Tin-Loi and Tseng (2003) for linear complementarity problems; nevertheless, the choice of the most reliable solution must involve the selection of which of them are directly reachable from the permanent loads only stress states set. In the 
companion paper (Part 2), Orduña and Lourenço (2005), a new solution technique is proposed that is capable to take the solution from a permanent loads stress state to a failure state under the variable loads action.

\section{Conclusions}

A three-dimensional limit analysis formulation for rigid block assemblages was presented. This formulation features limited compressive stresses, non-associated frictional sliding and torsion failures at the interfaces. The lack of theoretical work about the plastic torsion on frictional interfaces was faced and it was necessary to address theoretical developments in this subject, particularly focused on rectangular cohesionless interfaces. Also the interactions of the torsion strength with the shear forces and bending moments on the interface was addressed and a piecewise linear approximation proposed. This proposal permits to reduce a possible source of error in the three-dimensional limit analysis of structures modelled as rigid block assemblages.

The multiplicity of solutions to the limit analysis problem in presence of non-associated flow rules represents a challenge from a theoretical point of view. In the second part of this paper (Orduña and Lourenço, 2005) a novel solution procedure is proposed and a series of examples are given, aiming at validation of the proposed limit analysis model and solution procedure. The solution procedure follows, in an approximated way, the history of loading over the structure, for that reason has been named the load-path following procedure. This procedure provides better solutions than minimising the load factor and, moreover, it provides an insight into the structural behaviour along the loading history until failure with the same limited amount of information required by classic limit analysis.

\section{Acknowledgement}

The first author wishes to thank the scholarship made available to pursue his $\mathrm{PhD}$ studies by the Consejo Nacional de Ciencia y Tecnología of Mexico. The work was also partially supported by project SAPIENS 33935-99 funded by Fundação para a Ciência e Tecnologia of Portugal.

\section{References}

Baggio, C., Trovalusci, P., 1998. Limit analysis for no-tension and frictional three-dimensional discrete systems. Mech. Struct. Mach. 26 (3), 287-304.

Begg, D., Fishwick, R., 1995. Numerical analysis of rigid block structures including sliding. In: Middleton, J., Pande, G. (Eds.), Computer Methods in Structural Masonry, vol. 3, Portugal, pp. 177-183.

Casapulla, C., D'Ayala, D., 2001. Lower-bound approach to the limit analysis of 3D vaulted block masonry structures. In: Hughes, T.G., Pande, G.N. (Eds.), Computer Methods in Structural Masonry, vol. 5, Rome, Italy, pp. 177-183.

Ferris, M., Tin-Loi, F., 2001. Limit analysis of frictional block assemblies as a mathematical program with complementarity constraints. Int. J. Mech. Sci. 43, 209-224.

Goyal, S., Ruina, A., Papadopoulos, J., 1991. Planar sliding with dry friction. Part 1. Limit surface and moment function. Wear 143 (2), 307-330.

Grierson, D., 1977. Collapse load analysis. In: Cohn, M., Maier, G. (Eds.), Engineering Plasticity by Mathematical Programming. Pergamon Press, Univ. Waterloo, Canada, pp. 93-105.

Heyman, J., 1966. The stone skeleton. Int. J. Solids Struct. 2, 249-279.

Kamenjarzh, J., 1996. Limit Analysis of Solids and Structures. CRC.

Lourenço, P.B., Ramos, L.F., 2004. Characterization of the cyclic behavior of dry masonry joints. J. Struct. Eng. 130 (5), $779-786$.

Nadai, A., 1950. The problem of plastic torsion. Experimental representation of stress distribution, second ed. Theory of Flow and Fracture of Solids, vol. 1. McGraw-Hill, pp. 490-511 (Ch. 35).

Nielsen, M., 1999. Limit Analysis and Concrete Plasticity, second ed. CRC. 
Oliveira, D.V., 2003. Experimental and numerical analysis of blocky masonry structures under cyclic loading. Ph.D. thesis, University of Minho, Guimaraes, Portugal. Available from: <http://www.civil.uminho.pt/masonry $>$.

Orduña, A., 2003. Seismic assessment of ancient masonry structures by rigid blocks limit analysis. Ph.D. thesis, University of Minho, Guimaraes, Portugal. Available from: $<$ http://www.civil.uminho.pt/masonry $>$.

Orduña, A., Lourenço, P., 2003. Cap model for limit analysis and strengthening of masonry structures. J. Struct. Eng. 129 (10), 13671375.

Orduña, A., Lourenço, P.B., 2005. Three-dimensional limit analysis of rigid blocks assemblages. Part II: Load-path following solution procedure and validation. Int. J. Solids Struct., in press, doi:10.1016/j.ijsolstr.2005.02.011.

Tin-Loi, F., Tseng, P., 2003. Efficient computation of multiple solutions in quasibrittle fracture analysis. Comput. Methods Appl. Mech. Eng. 192 (11-12), 1377-1388.

Vasconcelos, G.F.M., Lourenço, P.B., 2004. Experimental assessment of the behaviour of unreinforced masonry walls subject to in plane cyclic actions (in Portuguese). In: 6th National Congress on Seismology and Seismic Engineering, Guimaraes Portugal, pp. 531-542. 